\title{
Fatores do bem-estar animal relacionados ao padrão da carne bovina: uma revisão
}

\author{
Animal Welfare Factors Related to Beef Standard: a review \\ Factores de bienestar animal relacionados con el estándar de carne de res: una revisión
}

\author{
Matheus Campos Castro \\ ORCID: https://orcid.org/0000-0002-9918-1491 \\ Universidade Estadual de Maringá, Brasil \\ E-mail: 1996mcastro@gmail.com \\ Eloize Silva Alves \\ ORCID: https://orcid.org/0000-0002-3340-8374 \\ Universidade Estadual de Maringá, Brasil \\ E-mail: eloizeetaus@gmail.com \\ Bruno Henrique Figueiredo Saqueti \\ ORCID: https://orcid.org/0000-0002-1118-4605 \\ Universidade Estadual de Maringá, Brasil \\ E-mail: bruno_saqueti@outlook.com \\ Jéssica Souza Alves \\ ORCID: https://orcid.org/0000-0002-3909-3617 \\ Universidade Estadual de Maringá, Brasil \\ E-mail: jessicasouza.uem@gmail.com \\ Joice Camila Martins Costa \\ ORCID: https://orcid.org/0000-0003-2175-7494 \\ Universidade Estadual de Maringá, Brasil \\ E-mail: joicecamilamart@gmail.com \\ Andressa Rafaella da Silva Bruni \\ ORCID: https://orcid.org/0000-0002-8236-1293 \\ Universidade Estadual de Maringá, Brasil \\ E-mail: rafaela_bruni@ hotmail.com \\ Zeinab El Hajj Hussein \\ ORCID: https://orcid.org/0000-0001-8700-0211 \\ Universidade Estadual de Maringá, Brasil \\ E-mail: zeinabnutri-209@outlook.com \\ Giovana Frigo \\ ORCID: https://orcid.org/0000-0002-1600-2102 \\ Universidade Estadual de Maringá, Brasil \\ E-mail: giovanafrigo@ hotmail.com \\ Oscar Oliveira Santos \\ ORCID: https://orcid.org/0000-0002-9631-8480 \\ Universidade Estadual de Maringá, Brasil \\ E-mail: oliveirasantos.oscardeoliveira@gmail.com \\ Jesui Vergilio Visentainer \\ ORCID: https://orcid.org/0000-0003-3412-897X \\ Universidade Estadual de Maringá, Brasil \\ E-mail: jesuiv@gmail.com
}

\begin{abstract}
Resumo
O presente estudo tem como objetivo fornecer uma compreensão sobre a relação das condições do bem-estar animal antecedentes ao abate à qualidade da carne baseada em uma revisão bibliográfica. Para isso foi realizado uma busca na literatura utilizando as seguintes palavras-chaves: Welfare, Animal Welfare, Pre-slaughter, Beef, Meat Quality. A partir do início do século XXI foi possível observar um crescente aumento de pesquisas pertencentes ao tema, isso acontece devido ao fato de que cada vez mais o bem-estar animal está relacionado ao estresse do animal, metabolismo durante o rigor mortis - responsável pela conversão do tecido muscular em carne de consumo, assim consequentemente os consumidores finais estão cada vez mais exigentes em relação a isso. No pós-abate de bovinos, podem surgir defeitos da qualidade da carne, denominadas como PSE (pálido, mole, exsudativo) e DFD (escuro, duro, seco), associados a um pH anormal da carne. O bem-estar animal está intimamente ligado desde a criação dos animais até a etapa de pré-abate da carne. A partir do estudo realizado, pode-se definir a necessidade de novas pesquisas que avaliem métodos de prevenção de estresse durante ao pré-abate.
\end{abstract}

Palavras-chave: Bem-estar animal; Rigor mortis; Carne bovina; Abate. 


\begin{abstract}
The present study aims to provide an understanding of the relationship between animal welfare conditions prior to slaughter and meat quality based on a literature review. For this, a literature search was carried out using the following keywords: Welfare, Animal Welfare, Pre-slaughter, Beef, Meat Quality. From the beginning of the 21st century it was possible to observe a growing increase in research on the subject, this is due to the fact that animal welfare is increasingly related to animal stress, metabolism during rigor mortis - responsible for the conversion of muscle tissue into consumable meat, so consequently end consumers are increasingly demanding in this regard. In the post-slaughter period of cattle, meat quality defects may appear, known as PSE (pale, soft, exudative) and DFD (dark, firm, dry), associated with an abnormal $\mathrm{pH}$ of the meat. Animal welfare is closely linked from the creation of animals to the preslaughter stage of meat. From the study carried out, it is possible to define the need for further research to assess methods of preventing stress during pre-slaughter.
\end{abstract}

Keywords: Animal welfare; Mortis rigor; Beef; Slaughter.

\title{
Resumen
}

El presente estudio tiene como objetivo proporcionar una comprensión de la relación entre las condiciones de bienestar animal antes del sacrificio y la calidad de la carne basándose en una revisión de la literatura. Para ello, se realizó una búsqueda bibliográfica utilizando las siguientes palabras clave: Bienestar, Bienestar Animal, Presacrificio, Carne vacuna, Calidad de la carne. Desde principios del siglo XXI se pudo observar un incremento creciente en la investigación sobre el tema, esto se debe a que el bienestar animal está cada vez más relacionado con el estrés animal, metabolismo durante el rigor mortis - responsable de la conversión del tejido muscular en carne consumible, por lo que los consumidores finales son cada vez más exigentes en este sentido. En el período posterior al sacrificio del ganado, pueden aparecer defectos en la calidad de la carne, conocidos como PSE (pálido, blando, exudativo) y DFD (oscuro, duro, seco), asociados a un pH anormal de la carne. El bienestar animal está estrechamente vinculado desde la creación de los animales hasta la etapa previa al sacrificio de la carne. A partir del estudio realizado, es posible definir la necesidad de realizar más investigaciones para evaluar los métodos de prevención del estrés durante el pre-sacrificio.

Palabras clave: Bienestar de los animales; Rigor mortis; Carne de res; Sacrificio.

\section{Introdução}

O bem-estar dos animais no abate afeta gradativamente a qualidade da carne, o que prejudica a segurança alimentar, e a economia na produção ou processamento de carne. Globalmente, milhares de doenças são transmitidas por alimentos e aproximadamente 420.000 mortes anuais têm sido atribuídas a patógenos microbianos, em grande parte devido a práticas de processamento de alimentos (assim como de carne) deficientes (Yenealem et al., 2020).

A qualidade da carne é relacionada as suas características sensoriais por fatores como a alimentação, manejo, raça e transporte (ante mortem) e condições de envelhecimento (tempo e temperatura), embalagem e cozimento, levando à variabilidade significativa (fatores post mortem) (Bonneau \& Lebret, 2010; Mcllveen \& Buchanan, 2001). Entre esses fatores, a raça é um dos mais importantes, mas diferentes sistemas de produção de gado e condições de manejo pré-abate, como transporte, tempo de espera, ruídos no matadouro ou mistura com indivíduos desconhecidos também são importantes (Ferguson \& Warner, 2008).

Em relação à cor da carne, as interações entre raça e sistema de produção pecuária e manejo pré-abate são significativas, consequentemente a interpretação dos principais efeitos é complexa (López-Pedrouso et al., 2020). Pode-se presumir que as condições na fazenda variam muito em muitos aspectos, o que também pode ter influenciado os níveis de estresse dos animais. Exemplos de fatores potencialmente influentes relacionados à fazenda são estratégias de criação, condições de alojamento e rotinas para o cuidado, alimentação e manejo dos animais, rotinas de pastejo e condições de saúde no rebanho (Hultgren et al., 2020).

Entre a ocorrência de locomoção da criação ao pré-abate, envolvem alguns problemas de bem-estar animal associados ao transporte quais podem ser evitados com o popular abate na fazenda, conforme relatado por Hultgren (2018). Existem opções de levar o matadouro para a fazenda e realizar o abate no local onde os animais foram criados, podendo assim evitar a exposição dos animais a ambientes desconhecidos, livre de estresse (Stocchi et al., 2014). 
Por outro lado, o abate industrial pode ter um maior potencial de investimentos para o manejo eficiente dos animais; o abate industrial é um local onde a tecnologia da máquina pode aumentar, em vez de aliviar, a pressão sobre os corpos que permanecem (Blanchette, 2020). Por outro lado, o abate industrial pode ter um maior potencial de investimentos para o manejo eficiente dos animais. Na verdade, pode ser difícil realizar o abate em espaços confinados em áreas remotas, com acesso limitado e confiável, além do controle e gasto de água potável e eletricidade (Greger, 2007). O presente estudo tem como objetivo fornecer uma compreensão profunda sobre a relação das condições do bem-estar animal antecedentes ao abate à qualidade da carne baseada em uma revisão bibliográfica.

\section{Metodologia}

Foram consultados em bases de dados de pesquisa como Google Scholar, Science direct e Web of Science utilizandose de palavras-chaves para busca: Welfare, Animal welfare, Pre-slaughter, Beef, Meat quality, sendo uma consulta metodológica qualitativa por revisão bibliográfica (Pereira et al., 2018).

\section{Resultados e Discussão}

\subsection{Coleta de dados}

Entre os resultados obtidos, foram encontrados um total de 164.120 trabalhos publicados, onde os dados se encontram apresentados na Figura 1:

Figura 1. Gráfico de publicações ao longo dos anos, utilizando as palavras-chave: Animal welfare, Pre-slaughter, Beef, Meat quality.

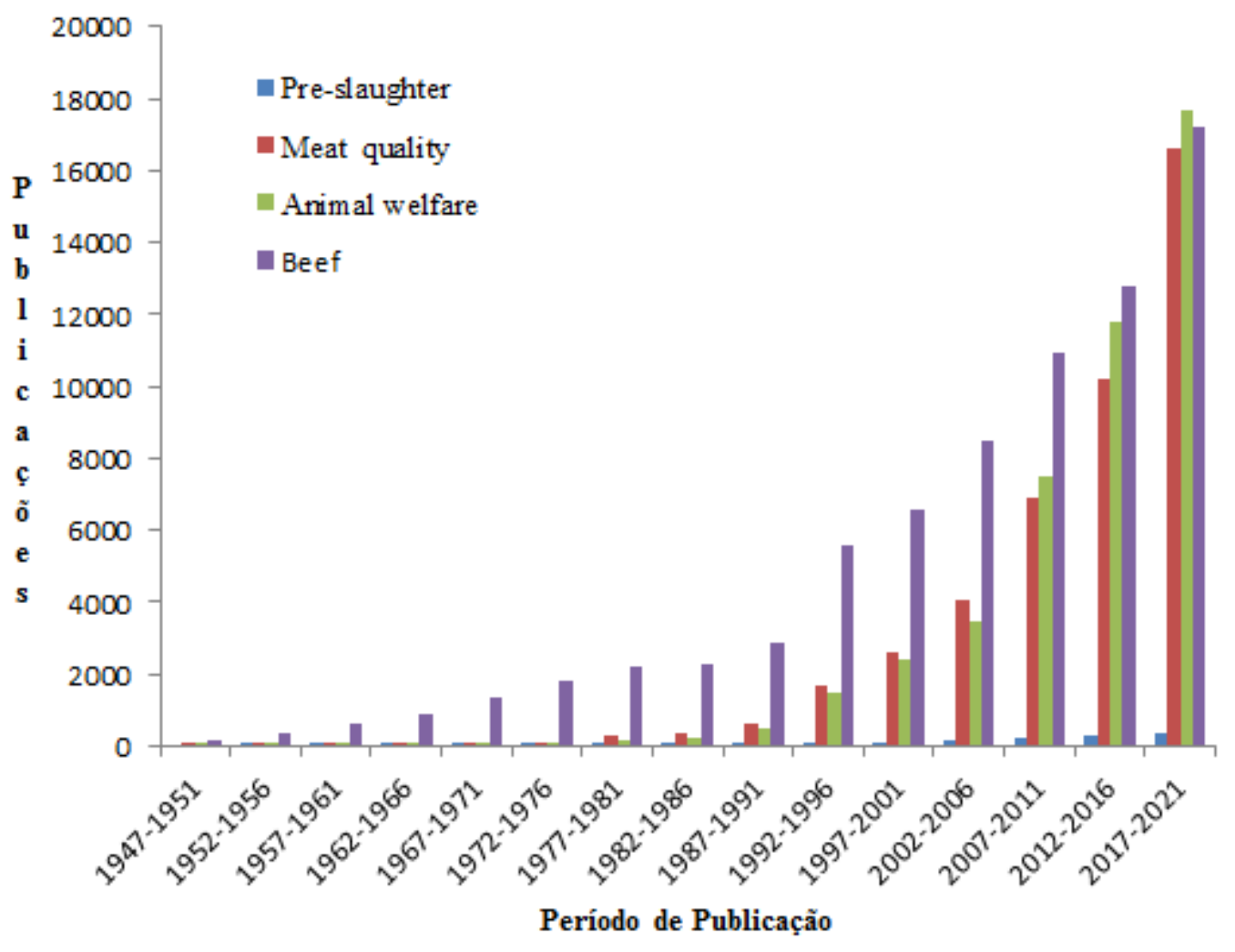

Fonte: Autores (2021) 
Analisando a Figura 1 foi possivel observar o crescente interesse por pesquisas a partir do inicio do seculo XXI utilizando dos temas abordados. Foi possivel notar um maior interesse conduzido pelo tema "beef" - carne, ao longo dos anos, fato esse que se deve ser estudado periodicamente para se ter uma maior qualidade do produto e seus derivados, contudo nos últimos 5 anos o tema mais pesquisado foi "animal welfare" - bem-estar animal, isso é de extrema importância, para o universo acâdemico se posicionar frente as novas descobertas, pois é fundamente diante a qualidade da carne.

\subsection{Bem-estar animal}

A importância atribuída ao bem-estar animal varia entre os países devido às diferenças nas tradições, demografia da pecuária, legislação, religião, educação, além do desenvolvimento econômico (Koknaroglu \& Akunal, 2013) e também entre os seus receptores (consumidores, varejistas, governos). No entanto, é o nível de interesse que o público em geral tem no bemestar animal que pode impulsionar a melhoria (Blokhuis et al., 2008; Veissier et al., 2008).

Além de levantar questões importantes, o bem-estar dos animais pode ter um impacto econômico significativo (Gallo \& Huertas, 2016). Devido a dificuldades inatas, o bem-estar no abate muitas vezes é definido em relação ao nível de estresse enfrentado pelo animal, qual seu conceito de estresse não possui uma definição clara, semelhante ao uso do termo "bem-estar" relacionado ao conceito de estresse (Wigham et al., 2018).

Em suma, a ciência do bem-estar animal ajuda a reavaliar os parâmetros das relações humanas com os animais mantidos. Fez isso, primeiro, trazendo a própria noção de bem-estar animal para uso comum (Rault et al., 2020). Além disso, alterou o conceito de bem-estar da simples remoção do sofrimento físico para um aprimoramento mais amplo. Criou o contexto para reafirmar o valor moral e econômico da vida animal mantida e, forneceu os meios para a sociedade definir os limites do que é considerado aceitável e do que não é, em nossas relações e tratamento com os animais (Buller et al., 2018).

\subsection{Melhoria do Sistema de produção}

Existem muitas tecnologias e práticas que afirmam resultar em bem-estar positivo, mas sem evidências fisiológicas e comportamentais do animal, essas alegações são válidas e não podem ser comparadas as tecnologias e práticas. O que é geralmente reconhecido é que avaliar apenas um indicador de bem-estar animal provavelmente não fornecerá uma compreensão completa das experiências do animal (Nicol et al., 2011).

Mesmo após executar mudanças de infraestrutura e possuir uma equipe treinada, podem haver custos contínuos associados a melhorias no bem-estar dos animais de criação. Por exemplo, qualquer intervenção para melhorar o bem-estar dos animais de fazenda podem exigir pessoal adicional contínuo, e pode haver custos contínuos associados aos suprimentos (Fernandes et al., 2021).

Os custos com a melhoria do sistema de produção devem ser todos incorporados ao custo do produto e, portanto, devem ser financiados pelos consumidores. Onde houver lacunas no conhecimento sobre o bem-estar dos animais de criação, é necessário investir em pesquisa, desenvolvimento e extensão do conhecimento (Agus \& Widi, 2018).

\subsection{Fatores que influenciam na qualidade da carne}

No pós-abate de bovinos, podem surgir defeitos da qualidade da carne, denominadas como PSE (Pale, Soft, Exudative) e DFD (Dark, Firm, Dry), associados a um pH anormal da carne. Isso geralmente acontece quando animais produtores de alimentos, antes do abate, estão sujeitos a más condições de bem-estar (Njisane \& Muchenje, 2017). Tais defeitos na carne apresentam efeitos prejudiciais, o que impede a lucratividade de produção de carne (Fernandes et al., 2021). 
Portanto, práticas cruéis contraditórias ao bem-estar animal durante o processo de abate devem ser proibidas. Considerando-se juntamente aos fatores de estresse quais podem aumentar a suscetibilidade de doenças em bovinos para abate, essas doenças podem ser transmissíveis aos humanos através da cadeia alimentar (Ianneti et al., 2020; Njoga et al., 2021).

Durante o rigor mortis, o pH do músculo diminui de 7 para aproximadamente 5,5; isso ocorre apenas por causa do metabolismo do glicogênio em ácido lático. Na Figura 2, está representado os defeitos que o metabolismo em ácido lático pode atingir e produzir defeitos na qualidade da carne.

Figura 2. Metabolismo do glicogênio em ácido lático durante o rigor mortis após 24 horas.

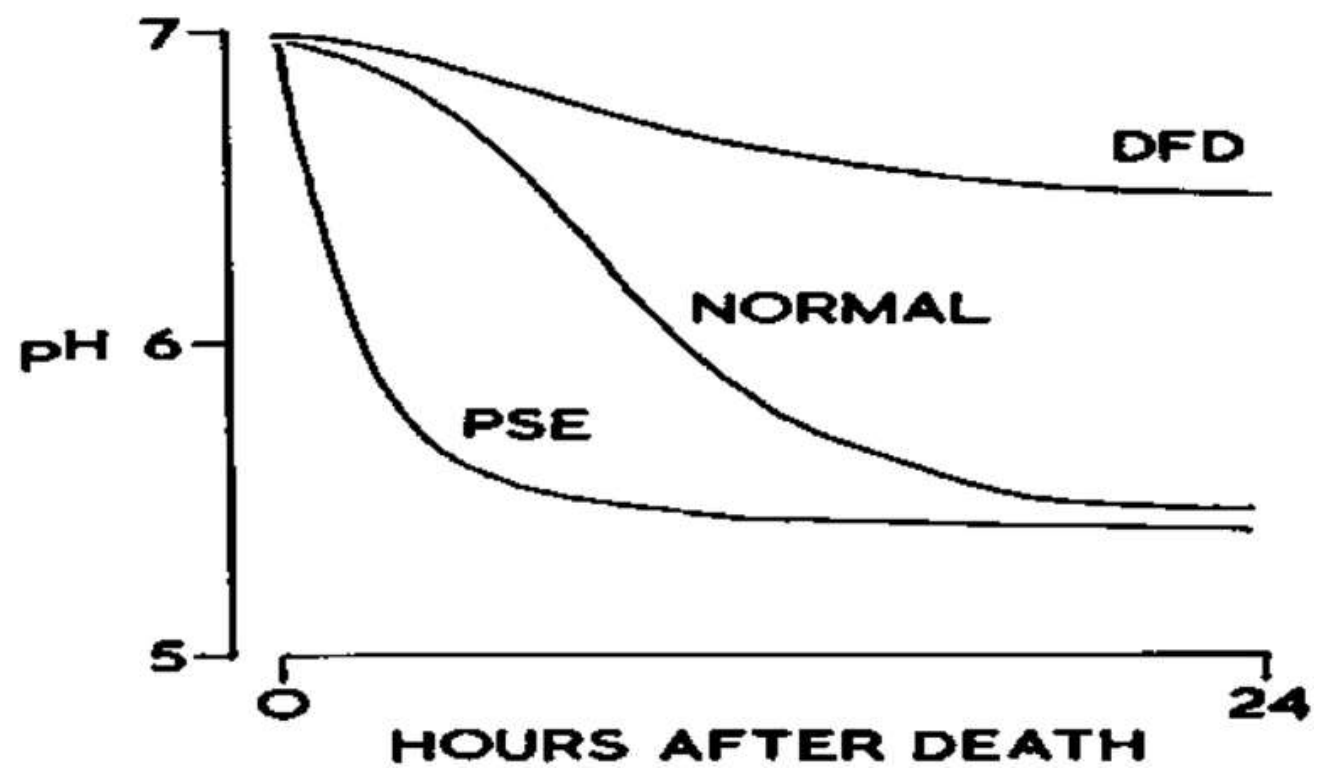

Fonte: Khan et al. (2018)

Essa redução do pH também é muito importante para a vida útil da carne produzida, porque a maioria das bactérias deteriorantes não pode crescer em $\mathrm{pH}$ baixo, sendo assim, o próprio ácido lático possui poder de agente antimicrobiano natural (Doores, 2005).

\subsection{Abate Halal}

O sistema de abate qual envolve percepção da religião, mais conhecidos pela literatura são o muçulmano, de acordo com Shariah lei Islâmica, possuem o abate Halal qual possui significado da palavra Halal por "permitido, autorizado"; em sua interpretação alguns requisitos de abate Halal devem ser atendidos (Miele, 2016):

- $\quad$ O animal deve estar são na hora do abate;

- O animal deve ser de espécie aceita para abate Halal;

- O equipamento de abate (lâmina) deve ser cirurgicamente afiada, isso deve cortar os principais vasos sanguíneos, a fim de assegure uma perda de sangue rápida e suficiente que conduza à morte;

- Deve ser concedido tempo suficiente para que o sangue fluindo escorrer para fora da carcaça;

- A pessoa que sangra o animal deve ser muçulmana,

- $\quad$ O abatedor deve recitar o nome de Deus em cada animal antes ou durante o corte do pescoço; 
Historicamente, os muçulmanos sempre abateram animais para consumo sem praticar atordoamento. Embora, seja um método altamente contencioso do ponto de vista do bem-estar animal moderno (Grandin, 2010), este método de abate ainda é muito difundido (Adzitey et al., 2011). Isso ocorre porque a maioria dos muçulmanos atribuem maior significado espiritual para a carne de animais abatidos desta forma (Farouk et al., 2014), provavelmente porque é o método que foi praticado pelo Profeta, Maomé.

O Islã como religião proíbe o abate de um animal na frente de outro animal, pois através dos sentidos ótico, olfativo e coclear os animais podem perceber o estado de estresse de seus compares, e produzir o estresse em ambos (Khan et al., 2018).

Para assegurar e impulsionar o crescimento da indústria de alimentos halal, a compreensão do comportamento de muçulmanos e não muçulmanos em relação aos alimentos halal. O crescimento da população muçulmana e a conscientização, juntamente com a aceitação e o aumento da popularidade dos alimentos halal entre os não muçulmanos, são as principais razões para o rápido crescimento do mercado de alimentos halal. No entanto, o compromisso com a religião não é a única razão para consumir alimentos halal. A certificação halal se torna o símbolo de limpeza e qualidade, e os clientes não muçulmanos consomem alimentos halal devido aos seus benefícios (Khan et al., 2020; Shahzad et al., 2020).

\section{Considerações Finais}

A partir da presente Revisão Bibliográfica realizada foi possível concluir que a o bem-estar animal é diretamente relacionado ao estresse animal, metabolismo durante o rigor mortis - responsável pela conversão do tecido muscular em carne de consumo e a qualidade final da carne. Visto que, o bem-estar animal é dado origem entre todo seu sistema de produção, desde a criação, até o momento final de sua captura, transporte e pré-abate. Novos estudos se tornam necessários para a avaliação de métodos de prevenção de estresse durante ao pré-abate.

\section{Agradecimentos}

Os autores agradecem ao Conselho Nacional de Desenvolvimento Científico e Tecnológico (CNPq) e Coordenação de Aperfeiçoamento de Pessoal de Nível Superior (CAPES) pelo auxílio financeiro.

\section{Referências}

Adzitey, F., Teye, G. A., \& Dinko, M. M. (2011). Pre and post-slaughter animal handling by butchers in the Bawku Municipality of the Upper East Region of Ghana. Livestock Research for Rural Development, 23, 1-8.

Agus, A., \& Widi, T. S. M. (2018). Current situation and future prospects for beef cattle production in Indonesia-A review. Asian-Australasian journal of animal sciences, 31,976 .

Blanchette, A. (2020). Porkopolis: American animality, standardized life, and the factory farm. Duke University Press Books.

Blokhuis, H. J., Keeling, L. J., Gavinelli, A., \& Serratosa, J. (2008). Animal welfare's impact on the food chain. Trends in Food Science \& Technology, 19, S79-S87.

Bonneau, M., \& Lebret, B. (2010). Production systems and influence on eating quality of pork. Meat science, 84, $293-300$.

Buller, H., Blokhuis, H., Jensen, P., \& Keeling, L. (2018). Towards farm animal welfare and sustainability. Animals, 8, 81.

Doores, S. (2005). Organic acids. Food Science and Technology-New York-Marcel Dekker, 145, 91.

Farouk, M. M., Al-Mazeedi, H. M., Sabow, A. B., Bekhit, A. E. D., Adeyemi, K. D., Sazili, A. Q., \& Ghani, A. (2014). Halal and kosher slaughter methods and meat quality: A review. Meat Science, 98, 505-519.

Ferguson, D. M., \& Warner, R. D. (2008). Have we underestimated the impact of pre-slaughter stress on meat quality in ruminants? Meat science, 80, $12-19$.

Fernandes, J. N., Hemsworth, P. H., Coleman, G. J., \& Tilbrook, A. J. (2021). Costs and Benefits of Improving Farm Animal Welfare. Agriculture, 11, 104.

Gallo, C. B., \& Huertas, S. M. (2016). Main animal welfare problems in ruminant livestock during preslaughter operations: a South American view. Animal, 10, 357-364. 
Grandin, T. (2010). Auditing animal welfare at slaughter plants. Meat Science, 86, 56-65.

Greger, M. (2007). The long haul: risks associated with livestock transport. Biosecurity and bioterrorism: biodefense strategy, practice, and science, 5, 301312 .

Hultgren, J. (2018). Is livestock transport a necessary practice? Mobile slaughter and on-farm stunning and killing before transport to slaughter. $C A B$ Reviews, 13, 1-15.

Hultgren, J., Segerkvist, K. A., Berg, C., Karlsson, A. H., \& Algers, B. (2020). Animal handling and stress-related behaviour at mobile slaughter of cattle. Preventive veterinary medicine, 177, 104959.

Iannetti, L., Neri, D., Santarelli, G. A., Cotturone, G., Vulpiani, M. P., Salini, R., \& Messori, S. (2020). Animal welfare and microbiological safety of poultry meat: Impact of different at-farm animal welfare levels on at-slaughterhouse Campylobacter and Salmonella contamination. Food Control, $109,106921$.

Khan, A., Mohammad, A. S., \& Muhammad, S. (2020). An integrated model of brand experience and brand love for halal brands: survey of halal fast food consumers in Malaysia. Journal of Islamic Marketing, 12, 1492-1520.

Khan, R., Guo, H., Raza, S. H. A., Rahman, A., Ayaz, M., \& Linsen, Z. (2018). Halal slaughtering, welfare, and empathy in farm animals: a review. Tropical animal health and production, 50, 1733-1738.

Koknaroglu, H., \& Akunal, T. (2013). Animal welfare: An animal science approach. Meat Science, 95, 821-827.

López-Pedrouso, M., Rodríguez-Vázquez, R., Purriños, L., Oliván, M., García-Torres, S., Sentandreu, M. Á., \& Franco, D. (2020). Sensory and physicochemical analysis of meat from bovine breeds in different livestock production systems, pre-slaughter handling conditions, and ageing time. Foods, 9 , 176.

McIlveen, H., \& Buchanan, J. (2001). The impact of sensory factors on beef purchase and consumption. Nutrition \& Food Science, 31, $286-292$.

Miele, M. (2016). Killing animals for food: How science, religion and technologies affect the public debate about religious slaughter. Food ethics, 1, 47-60.

Nicol, C. J., Caplen, G., Edgar, J., Richards, G., \& Browne, W. J. (2011). Relationships between multiple welfare indicators measured in individual chickens across different time periods and environments. Animal Welfare-The UFAW Journal, 20, 133.

Njisane, Y. Z., \& Muchenje, V. (2017). Farm to abattoir conditions, animal factors and their subsequent effects on cattle behavioural responses and beef quality-A review. Asian-Australasian journal of animal sciences, 30, 755.

Njoga, U. J., Njoga, E. O., Nwobi, O. C., Abonyi, F. O., Edeh, H. O., Ajibo, F. E., \& Guiné, R. P. (2021). Slaughter Conditions and Slaughtering of Pregnant Cows in Southeast Nigeria: Implications to Meat Quality, Food Safety and Security. Foods, 10, 1298.

Pereira, A. S., Shitsuka, D. M., Parreira, F. J., \& Shitsuka, R. (2018). Metodologia da pesquisa científica. [eBook]. Santa Maria. Ed. UAB / NTE / UFSM. https://repositorio.ufsm.br/bitstream/handle/1/15824/Lic_Computacao_Metodologia-Pesquisa-Cientifica.pdf?sequence=1 .

Rault, J. L., Waiblinger, S., Boivin, X., \& Hemsworth, P. (2020). The power of a positive human-animal relationship for animal welfare. Frontiers in Veterinary Science, 7, 857.Shahzad, M. A., Jun, D., Noor, G., \& Zubair, A. (2020). Causation of halal food consumption in China. Journal of Islamic Marketing, 7, 857.

Stocchi, R., Mandolini, N. A., Marinsalti, M., Cammertoni, N., Loschi, A. R., \& Rea, S. (2014). Animal welfare evaluation at a slaughterhouse for heavy pigs intended for processing. Italian journal of food safety, 3, 54-56.

Veissier, I., Butterworth, A., Bock, B., \& Roe, E. (2008). European approaches to ensure good animal welfare. Applied Animal Behaviour Science, 113, 279297.

Wigham, E. E., Butterworth, A., \& Wotton, S. (2018). Assessing cattle welfare at slaughter-Why is it important and what challenges are faced?. Meat science, $145,171-177$.

Yenealem, D. G., Yallew, W. W., \& Abdulmajid, S. (2020). Food safety practice and associated factors among meat handlers in Gondar Town: a CrossSectional. Journal of Environmental and Public Health, 2020, 1-7. 